\title{
Telepresence Mobile Robots Design and Control for Social Interaction
}

\author{
Tadele Belay Tuli ${ }^{1}\left[0 \cdot\right.$ Tesfaye Olana Terefe $^{2} \cdot$ Md Mamun Ur Rashid $^{3}$
}

Accepted: 30 June 2020 / Published online: 13 July 2020

(c) Springer Nature B.V. 2020

\begin{abstract}
Human-robot interaction has extended its application horizon to simplify how human beings interact with each other through a remotely controlled telepresence robot. The fast growth of communication technologies such as $4 \mathrm{G}$ and $5 \mathrm{G}$ has elevated the potential to establish stable audio-video-data transmission. However, human-robot physical interactions are still challenging regarding maneuverability, controllability, stability, drive layout, and autonomy. Hence, this paper presents a systematic design and control approach based on the customer's needs and expectations of telepresence mobile robots for social interactions. A system model and controller design are developed using the Lagrangian method and linear quadratic regulator, respectively, for different scenarios such as flat surface, inclined surface, and yaw (steering). The robot system is capable of traveling uphill $\left(30^{\circ}\right)$ and has a variable height $(600-1200 \mathrm{~mm})$. The robot is advantageous in developing countries to fill the skill gaps as well as for sharing knowledge and expertise using a virtual and mobile physical presence.
\end{abstract}

Keywords Telepresence mobile robot · Lagrangian model $\cdot$ LQR $\cdot$ QFD

\section{Introduction}

Today, mobile robots are capable of interacting with a human being in different environments. Mainly, the evolution of friendly robots, as well as the simplicity of controllers, makes robots to complement human in various activities. As we are in the digital era, smart systems are evolving daily to simplify human activities. Nowadays, there are humanoid robots that look like a human but with limited functionality. These robots are capable of executing a given task on behalf of

Electronic supplementary material The online version of this article (https://doi.org/10.1007/s12369-020-00676-3) contains

supplementary material, which is available to authorized users.

Tadele Belay Tuli

tadele.belay@aastu.edu.et

Tesfaye Olana Terefe

tesfitti2016@mtu.edu.et

Md Mamun Ur Rashid

mamun@niter.edu.bd

1 Department of Electromechanical Engineering, Addis Ababa Science and Technology University, Addis Ababa, Ethiopia

2 Department of Mechanical Engineering, Mizan Tepi University, Mizan Tepi, Ethiopia

3 Department of Electrical and Electronic Engineering, National Institute of Textile Engineering and Research (NITER), Dhaka 1350, Bangladesh the human being in the remote working space and hazardous environments.

Similarly, mobile robots are available for entertainment, audio-video based interaction, and remote monitoring. However, the social dimension and interaction level of humans and robots, particularly in the telepresence application, is not clear [15]. Telepresence robots still require improvements regarding autonomy, controllability, maneuverability, and stability to ensure safe interaction.

Currently, human social life is being dependent on technologies than ever, and it is complicated. Even though rapid progress has been achieved to address social and industrial issues using a remotely controlled working space, further investigation can be motivated by customer satisfaction and systematic requirement analysis. Telepresence robots, as intelligent and autonomous machines, could be a potential alternative to be included in the human social ecosystem. The first remote manipulator, which is implemented in 1948 [1], was the pioneer robot arm to imitate human activity using an electrically powered mechanical arm and joysticks to transfer motion. Such technologies are used in environments that are not accessible and hazardous to human workers [25]. Through time, remotely controlled robots widened their scope and application areas. Primarily, telepresence robots are used to bring individuals together through audio-video for social interaction. In this regard, mobility, bidirectional 
communication, and maneuverability are the required functionalities. Such functionalities are achieved using a display screen, web-based camera, audio systems, wheels, and power supply. According to [10], mobile telepresence robots are applied in office, health care, elder's place, and school environments.

The design of the telepresence robot that can travel and communicate with both audio and video transmission controlled from a remote is being considered as a physical presence. Some more specialized robots can perform services like cleaning and transportation of small objects. These robots communicate with the user using local networks like 3G or Wifi [23]. The robot is controlled through the remote controller connected to the device and to retrieve data. This environment is useful for the interaction of human beings using a remotely controlled mobile robot $[7,8,18,26]$. Augmented virtual reality is also applied to investigate human and telepresence robot's interaction behavior. Such approaches are useful to simulate the feeling of the human being in a human-robot interaction environment. In this regard, digital replication of the real-environment is being proposed by various research groups. Immersed virtual reality [24] makes the user to be immersed entirely and feel the digital environment and the users stimulation is used for design guidelines. Combining functionalities such as adaptable height [20], motion along the slope surface, and stability of the system along with low-speed control, is challenging [12]. Also, the traditional design guidelines for robots address safety and human-robot interaction, but it does not motivate how telepresence robots system design should be developed based on customer requirements $[14,27]$. The stability of the system can be regarded for bumping on the obstacle.

Customer-oriented product conceptual design is necessary to promote a systematic design in which the customer's preference and requirements are included. The quality function deployment (QFD) method is one of such approaches used to a couple of customer requirements to system design features. However, such methods are less applied for robot design and parameter selection. Various groups used QFD-based design approaches for autonomous underwater robot design [19], robot selection for specialized tasks [2], and assembly robot design evaluation [13]. In social environments, the customer's needs and expectations should guide the design, selection of parts, and system integration.

Hence, the current work aims to present a design and control approach for telepresence robots while investigating the interaction modalities based on customer requirements and expectation design guidelines. Here, the human-robot interaction is simulated, and the compatibility of the robot speed to a simple walk motion is investigated. Besides, the underlying safety issue when it comes to human-robot physical interaction was simulated using a physics solver. For this reason, the Unity3D game environment is implemented to visualize and simulate the scenario as a virtual reality environment. Accordingly, the current work shows the development of a human replicate that is capable of solving tremendous social and economic problems, including remote interaction, monitoring, social interdependence, human-like communication, and demonstration. In the approach, an insight on how customer requirements and market expectations define technical parameters and system design were presented. Such an approach is useful in designing a product that could fit the customers' expectations. Telepresence mobile robot technologies may support as a virtual presence to improve social interaction during crisis or pandemic, e.g., COVID-19. ${ }^{1}$

Similarly, the technology has been extended to serve in supermarkets, hotels, hospitals, schools, broadcasting stations, sports courts, parking's, banks, churches, and military yards. The telepresence mobile robot presented in the current work uses open source software and customized algorithms to transmit and receive audio and image data in 30 frames per second [12]. The modeling and design approach applied simplified modeling techniques such as two-dimensional models, customer requirement identification, and satisfaction analysis. Besides, the effect of the weight of the smart device attached at the top of the system for the overall structure is considered to be negligible.

\subsection{Requirement Analysis of Telepresence Robots}

The customer requirements were evaluated with their relative importance. Accordingly, 20 persons have participated in rating the relative importance of the design features. These requirements are listed based on functionalities and robot features design guidelines of classical robots, such as kinematics, dynamics, sensors, actuators, control, and robot programming [22]. The key features for the telepresence mobile robots are discussed below.

Handling/Maneuverability Handling and easy maneuvering are considered as an essential requirement in order to distinguish the system from stationary or uni-directional motions. In this regard, customers require to have a robot that can turn over itself in a minimum space, e.g., $0.5 \mathrm{~m}$. In mobile robots, two-wheel drive, four-wheel drive, or omnidirectional wheel drives are typical. Due to the advantage of regarding stability, low-speed control, and application environment, the two-wheel rear drive system is implemented. This particular design is known for easy handling and maneuverability using a rotating rear wheel. The front two wheels have a free degree of rotation about the vertical axis. The design has considered slope surfaces to verify that it does not turn over. The total

\footnotetext{
${ }^{1} \mathrm{https} / / / \mathrm{www}$. who.int/emergencies/diseases/novel-coronavirus2019.
} 
center of mass is considered at the minimum height again to confirm a precise motion further.

Controllable For a mobile robot that is characterized by movement, vision, and communication, the controllability of the overall system is necessary. This includes methods to control the audio-video communication channel, wheel driving system, and height adaptation. All these features are remotely controlled with the help of internet connection and PC or smartphone.

Easy to control at low speed The speed of the robot is controlled with the input voltage to the motor. The microcontroller limits the maximum and minimum speeds. The rest of the value of speed is defined by the user, depending on the mission of the robot. Unlike an inverted pendulum where the stability is entirely dependent on the speed of the robot, this design is less dependent on velocity for stability. Hence, it confirms the possibility of controlling the device at low speed.

Carry a user-interface device at $\mathbf{0 . 6} \mathbf{m}$ height For simplifying the usability and wide-range applicability, the robot is proposed to hold input systems at a flexibility height that rises from 300 to $1200 \mathrm{~mm}$. The user interface is designed to be accessible and understandable for all age peoples in different postures such as sitting, lying on the bed, and standing.

Robust (stable) Most of the application areas required for social interactions are not a smooth surface. The customers requested to use the robot in the living room, bedroom, and outdoor. In this area, most commonly, the floor is rough due to a furnished floor mattress. Therefore, such operating environments create higher resistance forces as well as easily obstruct the motion of the robot. Thus, the robustness and stability of the system is a regarded feature to avoid failure as well as to empower the robot traveling on various road types, whether it is on rough or obstacle. In this regard, this requirement is associated with the vibration, rigidity of the system, and wheel drives. Stability is essential to transfer a clear and noise-free video and sound. The placement of the chassis and all body on the smallest height from the ground strengthens the fact that the robot is not susceptible to turn over, severe vibrations, and resistance to external forces.

Compact A compact system with an extended capability owes the flexibility and usability of the system in various application ranges. Most existing telepresence robots have fixed dimensions though there are telescopic telepresence robots, hardly adaptable and agile. Ease to assemble, use, pack, and store are the other features considered to measure the compactness of the system.
Autonomy Autonomy is the primary requirement collected from the customer requirement information. The battery is the main problem of today's embedded system. Customers requirement shows a minimum of $10 \mathrm{~h}$ is required to operate autonomously without additional battery supply. Accordingly, the designed system consumes $23.28 \mathrm{Ah}$ in $12 \mathrm{~h}$. Therefore a 24,000 mAh battery supply is selected and considered during the hardware design. The battery supplies power for the embedded system and motors. If required, it is possible to power the computer/smartphone that is being used for audio-video controlling.

Dynamic performance Motion characteristics of the robot system determine how fast the robot travels, and the torque of the motors. In this regard, the dynamics of the robot is defined to be a maximum of $2 \mathrm{~m} / \mathrm{s}$. The primary source of motion is obtained from the two rear-wheels. In the same manner, the rear wheels are used to control the steering. The front wheels are free movers in any direction.

Layout 4 wheel Four wheels layout is used to share the distributed loads equally. Besides, this layout has contributed to achieving the desired characteristics like robustness, maneuverability, controllability, and stability.

According to the quality house and customer requirement analysis, engineering characteristics are determined based on the results obtained. Listed requirements are evaluated with respect to various parameters such as traveling speed, height, autonomy, required power, weight, slope angle, and the overall dimension. The correlation between the requirements and parameters are correlated using a correlation matrix. This correlation is classified into five categories. These are strong, medium, low, negative, and strong negative correlations (see Fig. 1).

The customer requirement analysis (refer Fig. 1), indicates the robustness or stability of the system is essential to allow the robot to move on rough roads and with the arguable slope. Stability provides clear and noise-free video and audio communication. Also, the placement of the chassis and all body on the smallest height from the ground strengthens the fact that the robot is not susceptible to turn over, severe vibrations, and resistance to external forces. The other approach we followed to evaluate this requirement feature is to assess the relevance of these features to the existing solutions, i.e., mobile telepresence robots. In this regard, six systems are systematically selected and analyzed (see Fig. 2).

The quality function house analysis shows that (refer Fig. 2), the design presented in this paper has an improved feature from some of the commercially existing mobile telepresence robots such as VGO, iRobot, Anybot QB, Mobi, Jazz, and Double R by about $21 \%$. 


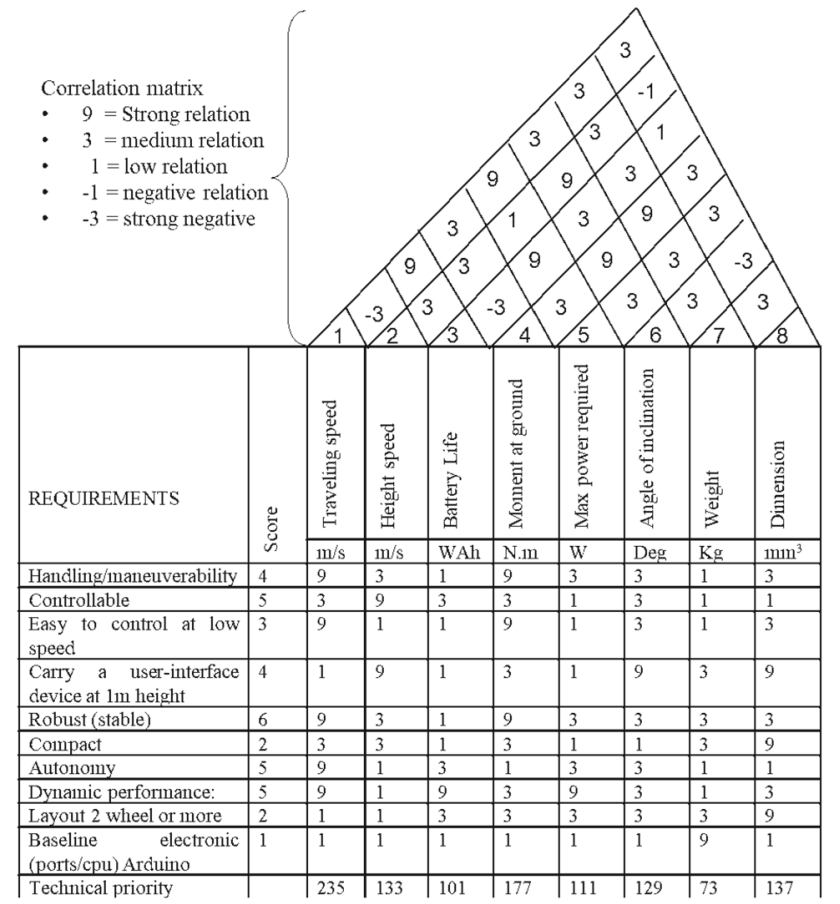

Fig. 1 Customer requirement analysis using quality house

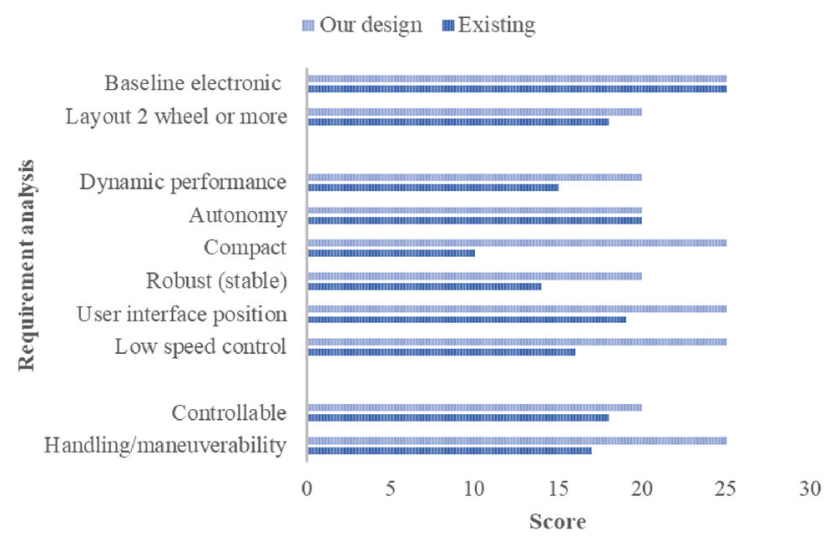

Fig. 2 Functional requirement analysis and feature comparison to commercially available systems

\subsection{Ethics of Robots for Social Interaction}

In the recent history of artificial intelligence and robotics, social and interactive skills of social intelligence robots are compulsory requirements in their several application areas where the robots want to work together and cooperate, and also with humans [3,6]. Robots are applied in a variety of areas because of their capacity and ability to work through human assisting or self-guidance. Social robots react to the environment by building a model of the world [11]. Some socially interactive robot responses from their human users because they track the procedures of behavior estimated by these human users [4]. In the collaboration of humans with a robot, the main problem is that robots can move very fast and can certainly injure a human operator, most likely in uncontrolled robot movements. So the question is, how can robots be made safer for the interaction of humans? The ethics of human and social robots can be obtained through the unique design, which involves simplifying the unidentified environments with humans for interacting with these robots. The human-robot interaction safety for social robots is one of the main areas of their ethics. The use of robot ethics is intensely linked to the way robots are professed. Nevertheless, ethics is not a prescriptive process; rather, it is a believed method. It is a virtual concept to stimulate a process of mechanism based on its environment [29]. When humans are around the robots, the conservative approach is to let them slow down the robot for the sake of safety. The efficiency and safety of robots affect robot behavior in their designs. Humans will respond to these behaviors, for interactions so that they will understand each other [21].

In human-robot interactions, people's interaction practices with robots substantially vary from people's practices interacting with most other technologies due to a strict social or sensitive component. In the media equation field of social robotics, the tendency to assign the level of intelligence and sociability affects the perceptions of peoples interacting with the robots. The human-like design and o the facial expressions encourage people to interact with the robot in a unique way, their autonomy. Technology should be built, designed, and made available only in so far it benefits humanity. There is no ethically consistent way resisting technological advances, therefore constant and complete ethics is required. The principles of robustness, fast reaction times, and context awareness are the outcomes of the development of safer robots for human-robot interaction [6].

Ethical input is vital at the design and execution stages of several modern-day technologies. Human-like interfaces, exploitation of human affective responses, robots, and other systems with intimate roles fall into this category.

\subsubsection{Significant Hazards and Usage Description}

The design of a telepresence robot is developed by identifying the possible potential hazards. Day to day human activity in dangerous places can be risky due to the over understanding or lack of knowledge about the robot's motion path. The failure in controlling software and hardware could lead to an unsafe environment if it is coupled with human interaction. Access by an unauthorized operator, failure due to mechanical system, the environmental consequence, improper setup, and the power systems are some of the risk conditions [9].

Although each of these potential hazards can be dangerous, they are each preventable as well as workers are well educated. The robot design can be used in many application areas. For supervision of humans of routine tasks: manufac- 


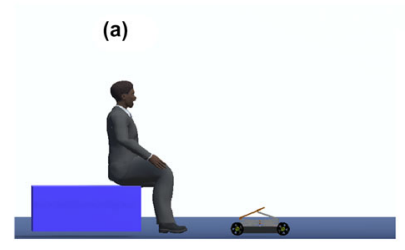

(c)

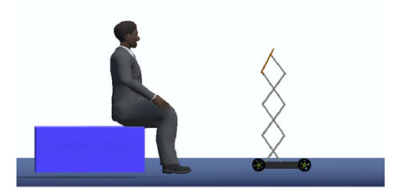

Fig. 3 Interaction scenarios

turing assembly lines, reading and sending of information, mail, and medicines in warehouses, offices, and hospitals. Remote-controlled environments, hazardous or inaccessible environments, undersea vehicles in entertainment areas providing teaching, comfort, assistance for children.

The design of this robot is limited to receive signals and deliver through the help of integrated smart technologies by audio-video transmission. In medicals applications, the use of this robot just supports overcoming the technical limits of conservative surgery executed by surgeons. In education, to maximize the students' learning outcomes in remote learning and to teach, it is essential to use the application of these telepresence robots in a teaching platform so that it can improve the credibility of the instructors. Further, it can also be used for students not able to go to school due to medical problems and other physical limitations. Particularly, a video conference can be one of the popular areas for telepresence based robots. To engage peoples easily office to office, between organizations and to work collaboratively in a condition at which peoples are not able to organize a physical event, we can use this robot. Moreover, safety instructions and guidelines described in the International Standard Organization (ISO) $13482^{2}$ is considered.

The other challenge of using telepresence robots is personal data under surveillance. In this regard, data privacy and use policy are required. In this regard, to comply with the national and international standards, the robot will be labeled for video surveillance to create awareness that peoples are being captured.

\subsection{Design Parameters and Technical Definitions}

The design parameters are inspired by a walking motion, such as speed, turning, and moment. The extended feature requirements are given in Table 1. The final dimension is selected after a careful geometric and kinematic analysis of

\footnotetext{
${ }^{2}$ https://www.iso.org/obp/ui/\#iso:std:iso:13482:ed-1:v1:en.
}

Table 1 Parameter definitions

\begin{tabular}{llll}
\hline Technical specifications & Unit & Min value & Max value \\
\hline Traveling speed & $\mathrm{m} / \mathrm{s}$ & 0 & 2 \\
Moment at ground & $\mathrm{Nm}$ & 0 & $0.85 \mathrm{~W}$ \\
Dimension & & & \\
$\quad$ Height & $\mathrm{cm}$ & 85 & 220 \\
Breadth & $\mathrm{cm}$ & 30 & 50 \\
Width & $\mathrm{cm}$ & 35 & 60 \\
Height speed & $\mathrm{m} / \mathrm{s}$ & 0 & 0.75 \\
Angle of inclination & $\mathrm{Deg}$ & 0 & 60 \\
Max power required & $\mathrm{kW}$ & 0 & 0.05 \\
Battery Life & $\mathrm{Ah}$ & 5 & 24 \\
Weight & $\mathrm{kg}$ & 5 & 15 \\
\hline
\end{tabular}

the system. The walking motion parameter considers an average walking speed, average human height, the average area required, and proper angle of inclination for slope surfaces.

\section{System Design}

The system is composed of android and window application tablets or smartphones with $4 \mathrm{G} / 5 \mathrm{G}$ or Wifi connectivity to allow access for broadcasting with the aid of webcam, microphone, and speaker of the device. The device and the embedded system can be connected using either Bluetooth or serial. Atmel 8-bit micro-controller is selected for controlling as LQR law. A 3D gyroscopic and inclination sensor is installed to control the velocity and height of the cylinder to avoid overturning on the inclined plane. This robot is characterized by movement, vision, speaking, and hearing features to allow audio-video communication. All these features are remotely controlled with the help of internet connection and PC or smartphone. The application software has a friendly graphic user interface to control speed, navigation, communication type, position adjustment, and connection query. The speed of the robot is controlled with the input voltage to the motor. The microcontroller limits the maximum and minimum speeds. The rest of the value of speed is defined by the user, depending on the mission of the robot. Unlike an inverted pendulum where the stability is entirely dependent on the speed of the robot, this design is less dependent on velocity for stability. Hence, it confirms the possibility of controlling the device at low speed.

\section{Mathematical Modeling and Control}

The mathematical models are used to simulate the system using symbolical computations. This approach is useful in 
analyzing the dynamics of the robot in a real-time scenario. In this regard, four main components, such as wheels, chassis, vertical link, and smart devices, are considered in the modeling procedure. In Fig. 4, the robot system is simplified into schematic representations, and the reference frames are attached to each link using Cartesian coordinate systems in order to measure the position of the robot relative to the global reference frame. The independent coordinate is represented on the longitudinal motion, $x(t)$, and the angular rotation, $\theta(t)$ of the wheel. The constraints applied come from no-slip on both lateral and longitudinal motion. Further simplifying, the $2 \mathrm{D}$ model is used for studying the longitudinal motion and turning over on the inclined surface. Here, the lateral motion is considered insignificant. The concentrated center of mass is selected at $R F 0$.

The possible constraint defined in the system is nonholonomic. Wheels are supposed to rotate without slipping, and as a result, the two wheels have a similar angular rotation.

$\phi=R \frac{d}{d t} \theta_{w}(t)=\frac{d}{d t} x(t)$

where $\phi$ is the steering angle, $\theta_{w}(t)$ is the wheel rotation angle and $x(t)$ is the longitudinal translation, and $R$ is radius the of the wheel.

In order to study the kinetics of the system, the NewtonEuler equation is used $[17,28]$. The traction forces and normal forces are derived from eliminating the unknown variables. The symbolic equation is used to study at varying parameters. Accordingly, the longitudinal and rotational velocities are given by Eqs. 2 and 3, respectively.

$$
\begin{aligned}
u(t) & =\frac{d}{d t} x(t) \\
u_{\theta}(t) & =\frac{d}{d t} \theta(t)
\end{aligned}
$$

Euler equation for the vertical body about $R F 0$ is used to consider if the load due to the upright body makes the whole system overturn with respect to the whole NewtonEuler equations. The simplified symbolic equation is given by Eq. 4 .

$$
m_{b} h \frac{d}{d t} u(t)+\frac{\left(2 m_{b} h^{2}\right)}{L} \frac{d}{d t} u_{\theta}(t)-m_{b} h \theta(t) g=0
$$

where $m_{b}$ is the lumped mass of the body, $\mathrm{h}$ is the position of the center of mass, $\mathrm{L}$ is the width of the car (i.e., along the $\mathrm{y}$ axis, refer Fig. 5), $u_{\theta}(t)$ is the angular velocity of the wheel, and $g$ is the gravitational force. The overall governing equation of the system is presented in Eq. 5, where $T c(t)$ is the external controlling torque, and $\mathrm{Me}$ represents the equivalent mass
$M_{e} \frac{d}{d t} u(t)+\mu \frac{d}{d t} u_{\theta}(t)-\frac{T c(t)}{R}+\psi \theta(t)=0$

where,

$\mu=\frac{\left(2 m_{b}\left(h^{2}+h R\right)\right)}{R L}$

and

$\psi=\left(\frac{\left(m_{b} h g\right)}{R}-\frac{\left(4 m_{b} h\left[u_{\theta}(t)\right]^{2}\right)}{L^{2}}\right)$

We create state-space matrix form representation using equation (1-5), and this system equations are applied to check the controllability, stability, and the response of the system [5].

$\dot{X}=A X+B U \quad Y=C X+D U$

Three scenarios are considered to analyze the response of the system based on Eq. 6. The first is to analyze the response of the system equation in normal conditions such as a flat surface with no rolling and side sliding effect. In this regard, the robot system is stable and controllable. In the second scenario, an inclined surface with variable heights is considered. In this case, the angle of inclinations $(\theta)$ shown in Fig. 5 are considered up to $30 \%$ of the slope. Similarly, back turning due to the gravity and height (h) of the robot support is one of the main challenges causing system instabilities. Therefore, the third scenario included the yaw motion, and the system stability and controllability are analyzed.

The Linear-Quadratic Regulator (LQR) design is selected for the system model given in Eq. 6. Specifically, we use the LQR method for determining our state-feedback control gain matrix. The matrix $\mathrm{R}$ and $\mathrm{Q}$ balance the relative importance of the control effort and error (deviation from 0 ), respectively, in the cost function of the system model. To start from the simple, assume $\mathrm{R}=1$, and $\mathrm{Q}=\mathrm{C}^{\prime} \mathrm{C}$ (see Eq. 8), which means to place equal importance on the control and the state variables, which are outputs of the mobile telepresence robot inclination angle and position of the robot. Essentially, the LQR-method allows for the control of both outputs. In this case, the controller can be tuned by changing the nonzero elements in the matrix to achieve the desired response. The system is verified whether the state is controllable or not. The rank of controllability and the length of the state matrix are equal. Hence, the state is controllable. At the same time, we can check the stability of the system looking into the Eigen-value. Since the Eigen-value has a positive real root, the system is not stable [16]. Hence it is necessary to stabilize using the LQR controller. The uncontrolled space state matrix is given by Eqs. 7 and 8 . 


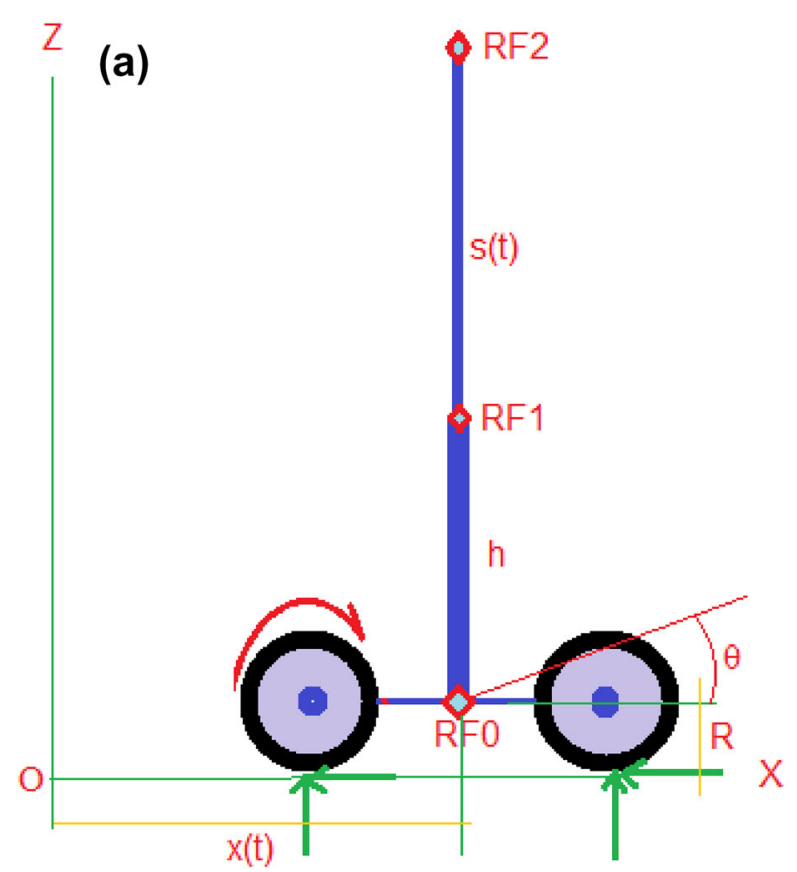

(b)

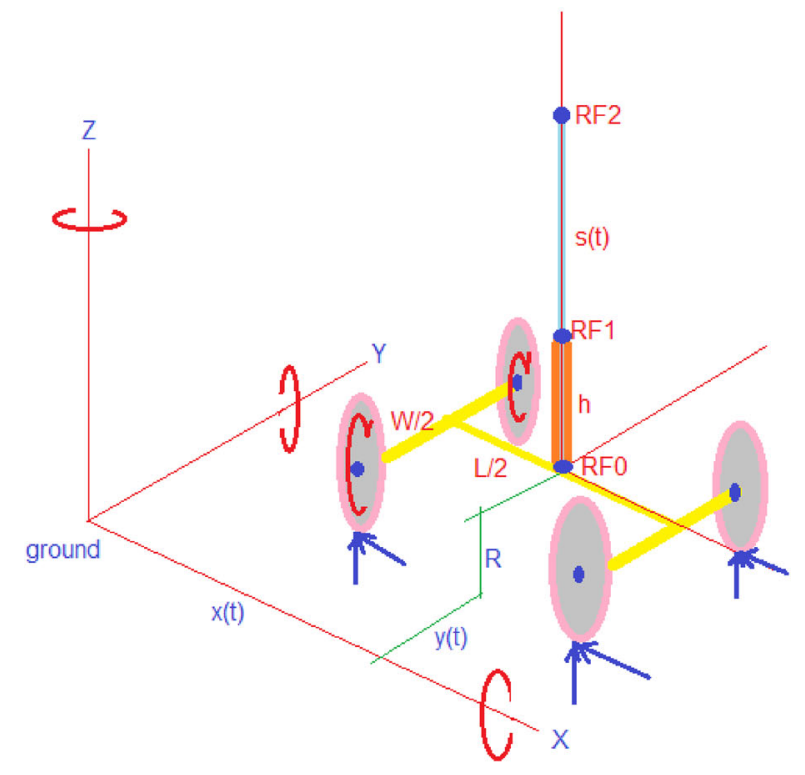

Fig. 4 Free-body schematic diagram a 2D view, b 3D view

$$
\begin{aligned}
A & =\left[\begin{array}{cccc}
0 & 1.0000 & 0 & 0 \\
0 & 0 & -24.7228 & 0 \\
0 & 0 & 0 & 5 \\
0 & 0 & 3.6350 & 0
\end{array}\right], \\
B & =\left[\begin{array}{cc}
0 \\
5.0403 \\
0 \\
-0.5306
\end{array}\right] \\
C & =\left[\begin{array}{llll}
1 & 0 & 0 & 0 \\
0 & 1 & 0 & 0 \\
0 & 0 & 1 & 0 \\
0 & 0 & 0 & 1
\end{array}\right], D=0
\end{aligned}
$$

The state space is controllable but not stable. The state matrix A has a zero and a positive real Eigen-value. Hence, the system is not stable. The LQR controller is used to control the state feedback matrix gain. The initial LQR-controller selected is given on Eq. 9.

$$
A=\left[\begin{array}{llll}
1 & 0 & 0 & 0 \\
0 & 1 & 0 & 0 \\
0 & 0 & 1 & 0 \\
0 & 0 & 0 & 1
\end{array}\right], R=[1]
$$

After introducing the LQR, the state is stabilized and is controllable. The state is improved in order to meet the required target in a better way (refer Eq. 10).

$$
A=\left[\begin{array}{llll}
4 & 0 & 0 & 0 \\
0 & 4 & 0 & 0 \\
0 & 0 & 1 & 0 \\
0 & 0 & 0 & 1
\end{array}\right], R=[1]
$$

The LQR controller-based improved space state matrices are given by Eq. 11, while the $\mathrm{C}$ and $\mathrm{D}$ matrix remains unchanged:

$A=\left[\begin{array}{cccc}0 & 1.0 & 0 & 0 \\ 10.1 & 20.4 & 161.8 & 351.7 \\ 0 & 0 & 0 & 5 \\ -1.1 & -2.2 & -16.0 & -37.0\end{array}\right], B=\left[\begin{array}{c}0 \\ 5.0 \\ 0 \\ -0.5\end{array}\right]$

The state responded with a time interval is simulated, and the simulation result is given in Figs. 5, 6 and 7. The simulation is conducted for linearized feedback control using the LQR controller with the initial and improved values. It is shown that in Fig. 6, before the improvement, the system takes about $45 \mathrm{~s}$ to be stable. However, the improved LQR matrix has changed this scenario to about $4 \mathrm{~s}$. This shows that our system has a stable state response in $4 \mathrm{~s}$. If we consider the angular motions (non-linear motions), it takes only $2 \mathrm{~s}$ to stabilize in rotational motions and steering systems (Fig. 5).

Torque is control input, and it is given as $\mathrm{Tc}(\mathrm{t})$ in Eq. 5. It transmits the user's desire into motion.

The telepresence communication device accepts motion commands from the remote and transcodes into the robot motion. This motion includes move forward, steer around, rise, and lower down the vertical skeleton. In this section, we 
(a)

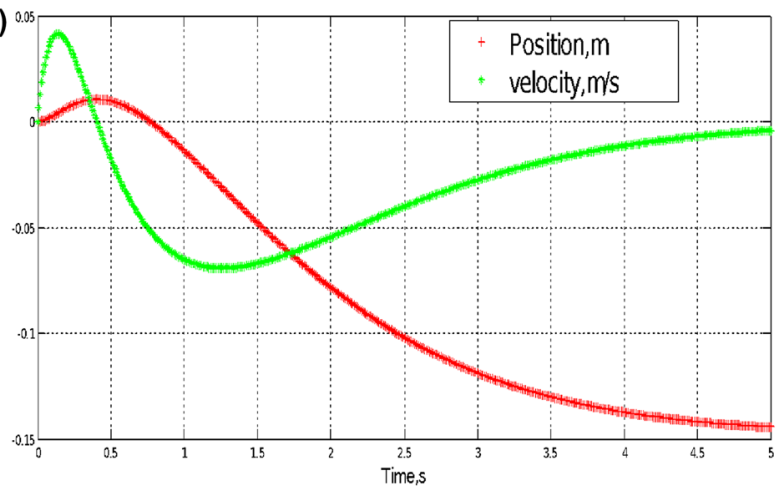

(b)

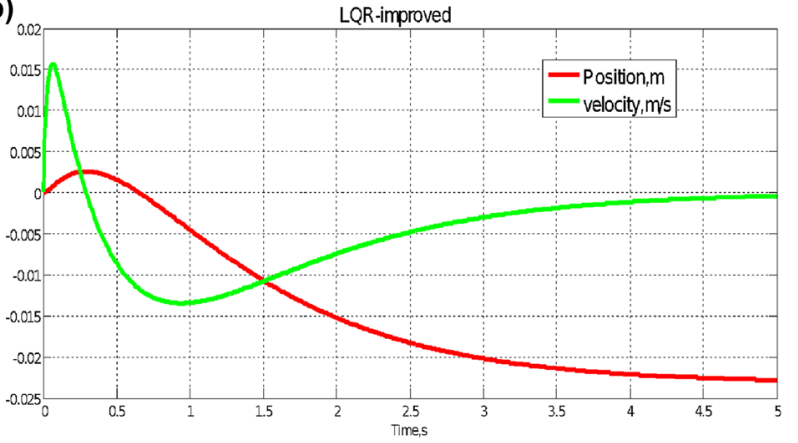

Fig. 5 Linear motion a without improvement, $\mathbf{b}$ with improvement on LQR
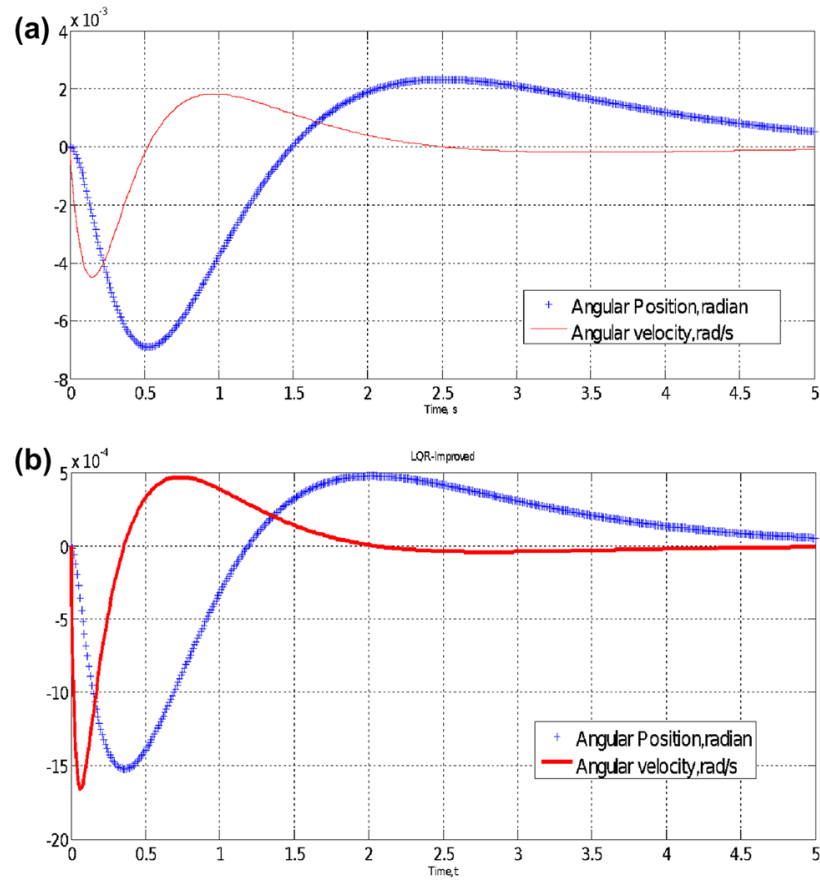

Fig. 6 Angular motion a without improvement, $\mathbf{b}$ with improvement on LQR

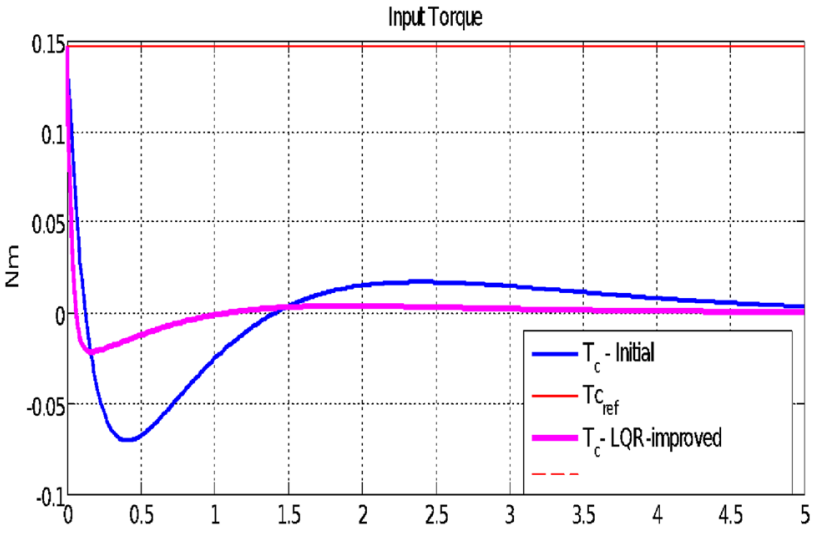

Fig. 7 Input torque response

consider only the torque response to the wheel motion and Fig. 7 gives the state behavior.

Finally, the mobile telepresence robot is designed using $3 \mathrm{D}$ tools and exported into a virtual simulator tool, which is a unity $3 \mathrm{D}^{3}$ game engine. The graphical simulation is implemented using real-time simulation (refer to Fig. 8). However, to simulate the interaction behavior of the human-robot - sitting position is selected to justify the usability and flexibility of the system. For the minimum robot position, it is required to readjust the robot height to the optimal position. Otherwise, there might be fatigue.

Similarly, a walk motion is applied to measure the speed and performance of the interaction. A walk motion is generated using physics in the Unity $3 \mathrm{D}$ environment. The robot model is simulated using a motion sequence that is synchronized with a human walk motion. In Fig. 9 a variable speed motion is demonstrated.

For this matter, a high-performance computer (32 GB RAM, Dell Precision laptop with NVidia graphics card) is used, and the result is promising in a way that, shortly, everybody can have his replicate at remote to carry out monitoring and surveillance.

\section{Conclusion and Future Outlook}

This paper is aimed to present a new solution and alternative modeling approach for mobile telepresence robots that are primarily targeted for social interaction and/or remote monitoring. The idea is to introduce physical and mobile human avatar or replica at low cost and robust design. Mainly, in developing countries where the skilled person is lacking, telepresence mobile robots could be used to remotely interact, communicate, and exchange skills with interactive and intuitive telepresence. Similarly, telepresence robots can be

\footnotetext{
${ }^{3}$ https://docs.unity3d.com/.
} 

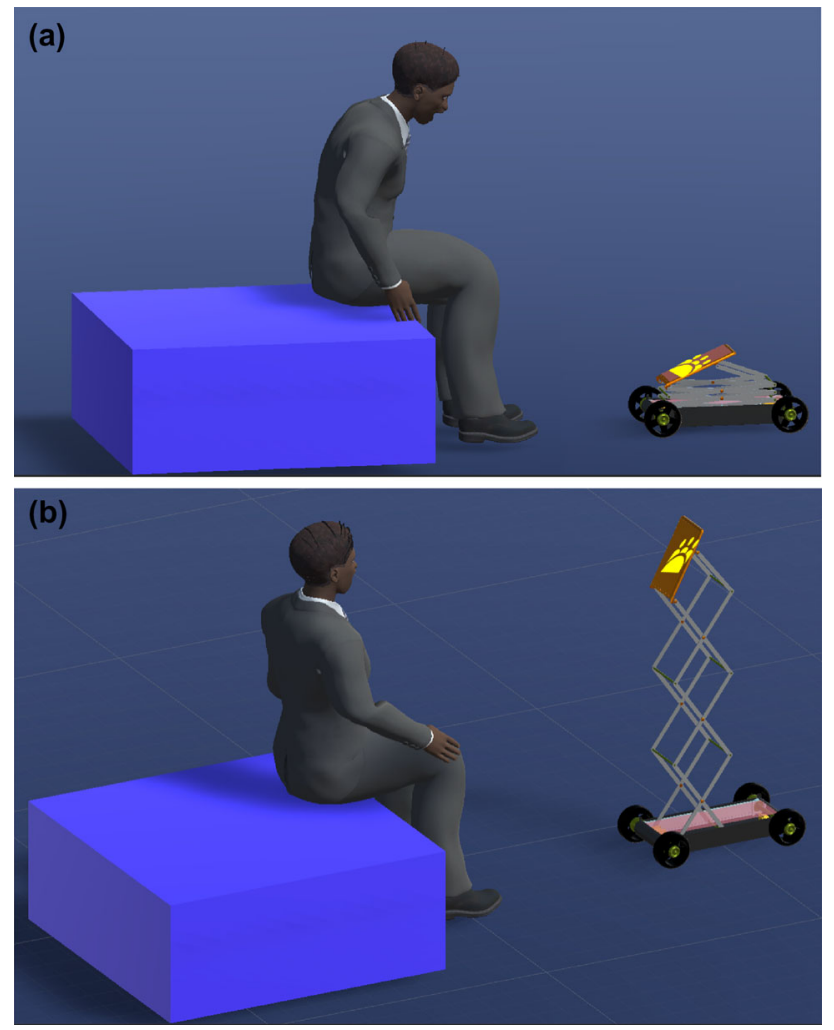

Fig. 8 Social interaction using telepresence robot a retracted position, b extracted position

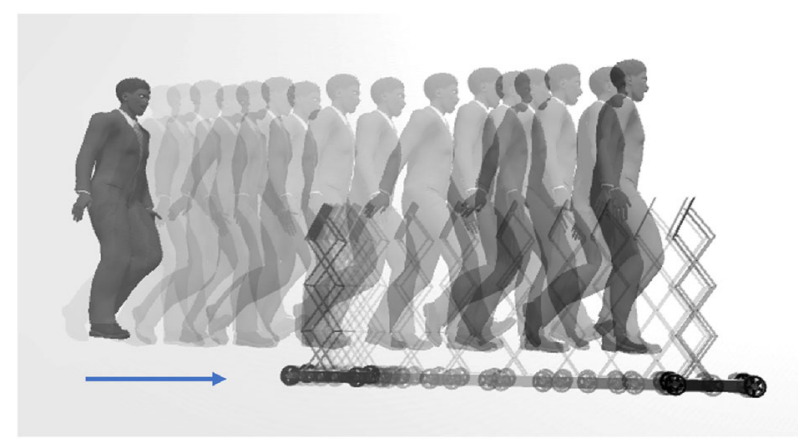

Fig. 9 Social interaction during walk motion

considered as an alternative physical presence on different social occasions as well as will be used to monitor the surroundings due to the mobility and autonomy of the robot. Today, the rapid evolution in imaging devices and real-time capable audio-video streaming capabilities through 4G/5G or Wifi elevates the potential of telepresence robots and their application areas. The challenges regarding maneuverability, controllability, stability, drive layout, and autonomy are addressed in this paper, primarily depending on the quality function and customers' requirements. These requirements are used to develop the engineering characteristics of mobile telepresence robots. Also, it is used to optimize controller parameters by considering the customer's expectation. In this regard, a system model and controller design are developed using the Lagrangian approach and linear quadratic regulator, respectively. Three different scenarios, such as flat surface, inclined surface, and yaw (steering) motion. The system and controller design are capable of traveling uphill of $30^{\circ}$ at a variable height of $600-1200 \mathrm{~mm}$. The 3D motion and system optimization will be considered in future works.

\section{Compliance with Ethical Standards}

Conflict of interest The authors declare that they have no conflict of interest.

\section{References}

1. Asada H, Slotine JJ, Slotine, JJE (1986) Robot analysis and control. Wiley. Google-Books-ID: KUG1VGkL3loC

2. Bhattacharya A, Sarkar* B, Mukherjee SK (2005) Integrating AHP with QFD for robot selection under requirement perspective. Int J Prod Res 43(17):3671-3685. https://doi.org/10.1080/ 00207540500137217

3. Dautenhahn K (2007) Socially intelligent robots: dimensions of human-robot interaction. Philos Trans R Soc B Biol Sci 362(1480):679-704. https://doi.org/10.1098/rstb.2006.2004

4. de Graaf MMA (2016) An ethical evaluation of human-robot relationships. Int J Soc Robot 8(4):589-598. https://doi.org/10.1007/ s12369-016-0368-5

5. Esfandiari RS, Lu B (2018) Modeling and analysis of dynamic systems. CRC Press, Boca Raton. https://doi.org/10.1201/b22138

6. Giuliani M, Lenz C, Müller T, Rickert M, Knoll A (2010) Design principles for safety in human-robot interaction. Int J Soc Robot 2(3):253-274. https://doi.org/10.1007/s12369-010-0052-0

7. Harmo P, Halme A, Pitkänen H, Virekoski P, Halinen M, Suomela J (2001) Moving eye-interactive telepresence over internet with a ball shaped mobile robot. IFAC Proc Vol 34(9):481-486. https:// doi.org/10.1016/S1474-6670(17)41754-X

8. Hiyama A, Kosugi A, Fukuda K, Kobayashi M, Hirose M (2017) Facilitating remote communication between senior communities with telepresence robots. In: Zhou J, Salvendy G (eds) Human aspects of IT for the aged population. Applications, services and contexts. Lecture notes in computer science. Springer, Cham, pp 501-515. https://doi.org/10.1007/978-3-319-58536-9_40

9. Kirschgens LA, Ugarte IZ, Uriarte EG, Rosas AM, Vilches VM (2019) Robot hazards: from safety to security. arXiv:1806.06681 [cs]

10. Kristoffersson A, Coradeschi S, Loutfi A (2013) A review of mobile robotic telepresence. Adv Hum-Comput Interact 2013:117. https://doi.org/10.1155/2013/902316

11. Lazzeri N, Mazzei D, Cominelli L, Cisternino A, De Rossi DE (2018) Designing the mind of a social robot. Appl Sci 8(2):302. https://doi.org/10.3390/app8020302

12. Lee H, Kim YH, Lee KK, Yoon DK, You BJ (2016) Designing the appearance of a telepresence robot, M4K: a case study. In: Koh JT, Dunstan BJ, Silvera-Tawil D, Velonaki M (eds) Cultural robotics. Lecture notes in computer science. Springer, Cham, pp 33-43. https://doi.org/10.1007/978-3-319-42945-8_3

13. Liao H, Chang Y, Wu D, Gou X (2019) Improved approach to quality function deployment based on Pythagorean fuzzy sets and application to assembly robot design evaluation. Front Eng Manag. https://doi.org/10.1007/s42524-019-0038-z 
14. Melendez-Fernandez F, Galindo C, Gonzalez-Jimenez J (2017) A web-based solution for robotic telepresence. Int J Adv Robot Syst 14(6). https://doi.org/10.1177/1729881417743738

15. Moniz AB, Krings BJ (2016) Robots working with humans or humans working with robots? Searching for social dimensions in new human-robot interaction in industry. Societies 6(3):23. https:// doi.org/10.3390/soc6030023

16. Nise NS (2004) Control systems engineering, 4th edn. Wiley, Hoboken

17. Ogata K (2014) System dynamics. Pearson education limited. OCLC: 1004510023

18. Osawa M, Imai M (2019) A robot for test bed aimed at improving telepresence system and evasion from discomfort stimuli by online learning. Int J Soc Robot. https://doi.org/10.1007/s12369019-00551-w

19. Pasawang T, Chatchanayuenyong T, Sa-ngiamvibool W (2015) QFD-based conceptual design of an autonomous underwater robot

20. Rae I, Takayama L, Mutlu B (2013) The influence of height in robot-mediated communication. In: 2013 8th ACM/IEEE international conference on human-robot interaction (HRI), pp 1-8. https://doi.org/10.1109/HRI.2013.6483495. ISSN: 2167-2148

21. Rodogno R (2016) Ethics and social robotics. Ethics Inf Technol 18(4):241-242. https://doi.org/10.1007/s10676-016-9412-2

22. Siciliano B, Khatib O (eds) (2016) Springer handbook of robotics, 2nd edn. Springer Handbooks. Springer

23. Tan Q, Denojean-Mairet M, Wang H, Zhang X, Pivot FC, Treu R (2019) Toward a telepresence robot empowered smart lab. Smart Learn Environ 6(1):5. https://doi.org/10.1186/s40561-019-00843

24. Tan Z, Hu Y, Xu K (2017) Virtual reality based immersive telepresence system for remote conversation and collaboration. In: Chang J, Zhang JJ, Magnenat Thalmann N, Hu SM, Tong R, Wang W (eds) Next generation computer animation techniques. Lecture notes in computer science. Springer, Berlin, pp 234-247

25. Trevelyan JP, Kang SC, Hamel WR (2008) Robotics in hazardous applications. In: Siciliano B, Khatib O (eds) Springer handbook of robotics. Springer, Berlin, pp 1101-1126. https://doi.org/10.1007/ 978-3-540-30301-5_49

26. Tsai TC, Hsu YL, Ma AI, King T, Wu CH (2007) Developing a telepresence robot for interpersonal communication with the elderly in a home environment. https://doi.org/10.1089/tmj.2006. 0068

27. Tsui KM, Yanco HA (2013) Design challenges and guidelines for social interaction using mobile telepresence robots. Rev Hum Factors Ergon 9(1):227-301. https://doi.org/10.1177/ $1557234 X 13502462$
28. Tuli TB (2018) Mathematical modeling and dynamic simulation of gantry robot using bond graph. In: Mekuria F, Nigussie EE, Dargie W, Edward M, Tegegne T (eds) Information and communication technology for development for Africa. Lecture notes of the institute for computer sciences, social informatics and telecommunications engineering. Springer, Cham, pp 228-237. https://doi. org/10.1007/978-3-319-95153-9_22

29. Yigit T, Kose U, Sengoz N (2018) Robotics rights and ethics rules. J Multidiscip Dev 3(1):30-37

Publisher's Note Springer Nature remains neutral with regard to jurisdictional claims in published maps and institutional affiliations.

Tadele Belay Tuli received B.Sc. in Mechanical Engineering, M.Sc. in Manufacturing Engineering, M.Sc. in Mechatronics Engineering from Bahir Dar University, Adama Science and Technology University, Ethiopia and University of Trento, Italy in 2008, 2012, 2015 respectively. He is a faculty member of Addis Ababa Science and Technology University, Ethiopia. Currently, he is pursuing his Ph.D. at the University of Siegen, Germany. His research interests include real-time robot control, motion tracking and modeling, human-robot collaboration, and sensor integration.

Tesfaye Olana Terefe currently works at the Mechanical Engineering, Mizan-Tepi University. He received his B.Sc. in Mechanical Engineering and M.Sc. in Mechanical Design from Jimma University in 2018. Now, his research area focuses on Mechanical Design for Industrial applications, including locomotive robots for human interaction.

Md Mamun Ur Rashid received B.Sc. in Electrical \& Electronic Engineering from Dhaka University of Engineering and Technology (DUET), Bangladesh in 2007, and an M.Sc. in Telecommunications Engineering from the University of Trento, Italy in 2017. Currently, he is an Assistant Professor and Head, Department of Electrical \& Electronic Engineering at National Institute of Textile Engineering and Research (NITER), Bangladesh. His research interests include smart grids, energy optimization, renewable energy, and robotic engineering. 